\title{
Impacto da pandemia de COVID-19 no atendimento do serviço de radioterapia em um hospital público de Salvador/BA
}

\author{
Impact of the COVID-19 pandemic on the service of radiotherapy in a public hospital \\ in Salvador/BA
}

\author{
Andressa Souza Jesus ${ }^{1 *}$, Tamires Dos Santos Guedes², Gabriela Botelho Martins ${ }^{3}$ \\ ${ }^{1}$ Fisioterapeuta. Mestranda do Programa de Pós-Graduação em Processos Interativos dos Órgãos e Sistemas, \\ Instituto de Ciências da Saúde, Universidade Federal da Bahia-ICS/UFBA. ${ }^{2}$ Enfermeira da Unidade de Alta \\ Complexidade em Oncologia Nossa Senhora de Fátima, Obras Sociais Irmã Dulce, Salvador, BA. ${ }^{3}$ Professora Adjunto \\ do Programa de Pós-graduação em Processos Interativos dos Órgãos e Sistemas, ICS/UFBA.
}

\begin{abstract}
Resumo
Introdução: o câncer é uma das principais causas de morte no mundo e o seu tratamento, associado com o processo da doença, acaba tornando esses pacientes mais vulneráveis. A pandemia de COVID-19 foi declarada no Brasil em março de 2020 e se estende até o presente momento, com centenas de milhares de infectados e de óbitos. No seu início, algumas medidas de prevenção foram adotadas, principalmente para populações de risco como os pacientes oncológicos, mediante o fechamento de centros de diagnósticos e de serviços eletivos, além da aderência ao distanciamento social. Objetivo: este estudo teve como objetivo determinar o impacto quantitativo gerado no atendimento de pacientes em um serviço de radioterapia, no perfil sociodemográfico dos pacientes oncológicos e no estadiamento tumoral desses pacientes. Metodologia: Tratou-se de estudo observacional descritivo, onde foram coletados dados dos prontuários dos pacientes, a partir do relatório mensal de atendimentos iniciais do serviço de radioterapia de uma Unidade de Alta Complexidade em Oncologia, em hospital público de Salvador, referentes às terapias iniciadas no período entre março e agosto dos anos de 2019 e 2020. Resultados: avaliaram-se 259 prontuários no ano de 2019 e 227 em 2020 . A maioria dos pacientes foi do sexo feminino (78\%) nos dois anos e residentes da cidade de Salvador. Segundo os resultados obtidos, percebeu-se uma diminuição de $12 \%$ no número de atendimentos realizados em 2020 , com uma piora no estadiamento tumoral desses pacientes, chegando a um aumento de $115 \%$ nos casos de presença de metástase. As principais neoplasias encontradas foram câncer de mama, cabeça e pescoço, próstata e colo do útero. Conclusões: houve uma redução no número de atendimentos na referida unidade, não existindo grande mudança no perfil desses pacientes, que apresentam uma amostra predominantemente do sexo feminino, sendo sua maioria residente da cidade de Salvador-BA. Porém foi possível notar uma diminuição da população acima de 60 anos e também um agravamento do estadiamento tumoral.
\end{abstract}

Palavras-chaves: Estadiamento de neoplasias. Instituições de oncologia. Neoplasias malignas. COVID-19.

\begin{abstract}
Introduction: Cancer is one of the main causes of death in the world and its treatment, associated with the disease process, ends up making these patients more vulnerable. The COVID-19 pandemic was declared in Brazil in March 2020 and has continued to date, with hundreds of thousands of infected people and deaths. At its beginning, some prevention measures were adopted, mainly for populations at risk such as cancer patients, through the closing of diagnostic centers and elective services, in addition to adherence to social distance. Objective: this study aimed to determine the quantitative impact generated on the care of patients in a radiotherapy service, on the sociodemographic profile of cancer patients and on the tumor staging of these patients. Methods: this was a descriptive observational study, where data were collected from the patients' medical records, from the monthly report of initial care at the radiotherapy service of a High Complexity Oncology Unit, in a public hospital in Salvador, regarding the therapies started in the period between March and August in the years 2019 and 2020. Results: two hundred and fifty nine (259) medical records were evaluated in 2019 and 227 in 2020. Most patients were female (78\%) in both years and residents in the city of Salvador. According to the results obtained, there was a 12\% decrease in the number of consultations performed in 2020, with a worsening in the tumor staging of these patients, reaching an increase of $115 \%$ in cases of metastasis. The main neoplasms found were breast, head and neck, prostate and cervical cancer. Conclusions: there was a reduction in the number of consultations in that unit, with no major change in the profile of these patients, who have a predominantly female sample, most of them residing in the city of Salvador-BA. However, it was possible to notice a decrease in the population over 60 years of age and also an aggravation of tumor staging.

Keywords: Staging of Neoplasms. Oncology Institutions. Malignant Neoplasms. COVID-19.
\end{abstract}

\section{INTRODUÇÃO}

Correspondente/Corresponding: *Andressa Souza Jesus - Instituto de Ciências da Saúde, Laboratório de Bioquímica Oral, Sala 413. - End: Av. Reitor Miguel Calmon, s/n, Vale do Canela - 40.110-100 Salvador, BA. -Tel: +55 71 3283-8910. -E-mail: andressasouzajesus@hotmail.com
No dia 9 de janeiro de 2020, foi reportado pela World Health Organization (WHO) que o surto de pneumonia viral que atingiu a China foi ocasionado por um novo coronavírus (Sars-Cov-2), sendo declarada em 30 de janeiro do mesmo ano como uma emergência de saúde pública 
de interesse internacional. Devido aos níveis alarmantes de contaminação e grau de severidade apresentada, a COVID-19 foi caracterizada com uma pandemia no dia 11de março de 2020 (WHO, 2021a).

Até o dia 25 de setembro de 2021, o número de casos confirmados de pessoas infectadas pelo novo coronavírus no Brasil era de $21,283,567$ milhões de casos, totalizando até essa mesma data, 592,316 mil mortes (WHO, 2021a). Mais de um ano depois a situação ainda se estende de forma grave.

Mesmo com estudos revelando uma maior susceptibilidade de pacientes oncológicos de serem infectados pelo novo coronavírus e apresentarem um risco maior de desenvolver formas mais severas da doença e até morrer (TIAN et al., 2020; ZHANG et al., 2020), atendimentos em hospitais de cuidado ao paciente oncológico, casas de cuidados paliativos e hospices se mantiveram abertos garantindo o tratamento desses pacientes, enquanto muitos centros de diagnóstico e acompanhamento tiveram suas atividades paralisadas. Outras medidas como suspensão de visitas, adesão de consultas pelo telefone ou chamada de vídeo foram adotadas, visando diminuir os riscos de contaminação (CHAN et al., 2020).

O câncer é uma das principais causas de mortes no mundo (WHO, 2021b), sendo a responsável por cerca de 9.958,133 mortes no ano de 2020 (INTERNATIONAL AGENCY FOR RESEARCH ON CANCER, 2020). Segundo Fialka-Mose et al. (2003), com o avanço médico no tratamento do câncer, o uso combinado de procedimentos cirúrgicos, quimioterapia e radioterapia, o número de pacientes que sobrevivem às neoplasias malignas é superior à $50 \%$. Porém, o processo da doença, juntamente com as terapias médicas, pode gerar várias complicações, limitando a atividade e a participação dos pacientes oncológicos nas atividades de vida diária (SANTIAGO-PALMA; PAYNE, 2001; FIALKA-MOSER et al., 2003).

Com todas as modificações na rotina de diagnóstico e tratamento do paciente oncológico, bem como o medo pela infecção, que levou muitos pacientes a adiarem a ida até os centros especializados, não se sabe como a pandemia pelo COVID-19 pode ter impactado no atraso desses diagnósticos, no agravamento da condição clínica geral, bem como no encaminhamento para tratamento.

Sendo assim, este estudo teve como objetivo determinar o impacto quantitativo gerado no atendimento de pacientes em um serviço de radioterapia, no perfil socio- demográfico dos pacientes oncológicos e no estadiamento tumoral durante os primeiros seis meses da pandemia de COVID-19, cujo início ocorreu no começo de 2020 e se estende até o momento atual (setembro de 2021).

\section{METODOLOGIA}

Trata-se de um estudo observacional descritivo, utilizando uma amostra não probabilística de conveniência, onde foram incluídos todos os prontuários dos pacientes que constavam no relatório mensal de atendimento de pacientes iniciais do serviço de radioterapia de uma Unidade de Alta Complexidade em Oncologia, de um hospital público de Salvador, referentes às terapias iniciadas no período entre março e agosto dos anos de 2019 e 2020..

Por tratar-se de uma pesquisa envolvendo seres humanos, mesmo que de forma indireta, o projeto foi submetido à avaliação e aprovação pelo Comitê de Ética em Pesquisa da Instituição, tendo sido aprovado pelo CAAE no 46328921.0.0000.0047, respeitando a Resolução no 466/12 do Conselho Nacional de Saúde.

As coletas de dados foram feitas mediante análise dos prontuários, incluindo as seguintes informações: sexo, idade, se reside em Salvador (capital) ou região metropolitana, ou se faz tratamento fora do domicílio (interior do Estado), data da consulta inicial para realização de radioterapia e data de realização da primeira sessão de radioterapia, estadiamento dos tumores malignos e os tipos de tumores atendidos no período já referido.

Os dados obtidos foram tabulados com estatística descritiva, com uso de médias e percentuais, e posteriormente expostos em tabelas e gráficos, utilizando-se o programa Excel da Microsoft Office 365.

\section{RESULTADOS}

O fluxo dos prontuários selecionados para a pesquisa está representado no fluxograma abaixo (Figura 1). Foram excluídos da pesquisa prontuários repetidos nas listas, os que não contavam com folha de atendimento do serviço de radioterapia ou apresentavam folha em branco ou incompletas.

Os dados sociodemográficos dos pacientes, coletados a partir dos prontuários disponíveis, estão registrados na Tabela 1. O número de atendimentos mensal para cada ano estudado está presente na Figura 2. 
Figura 1 - Fluxograma da seleção de prontuários.

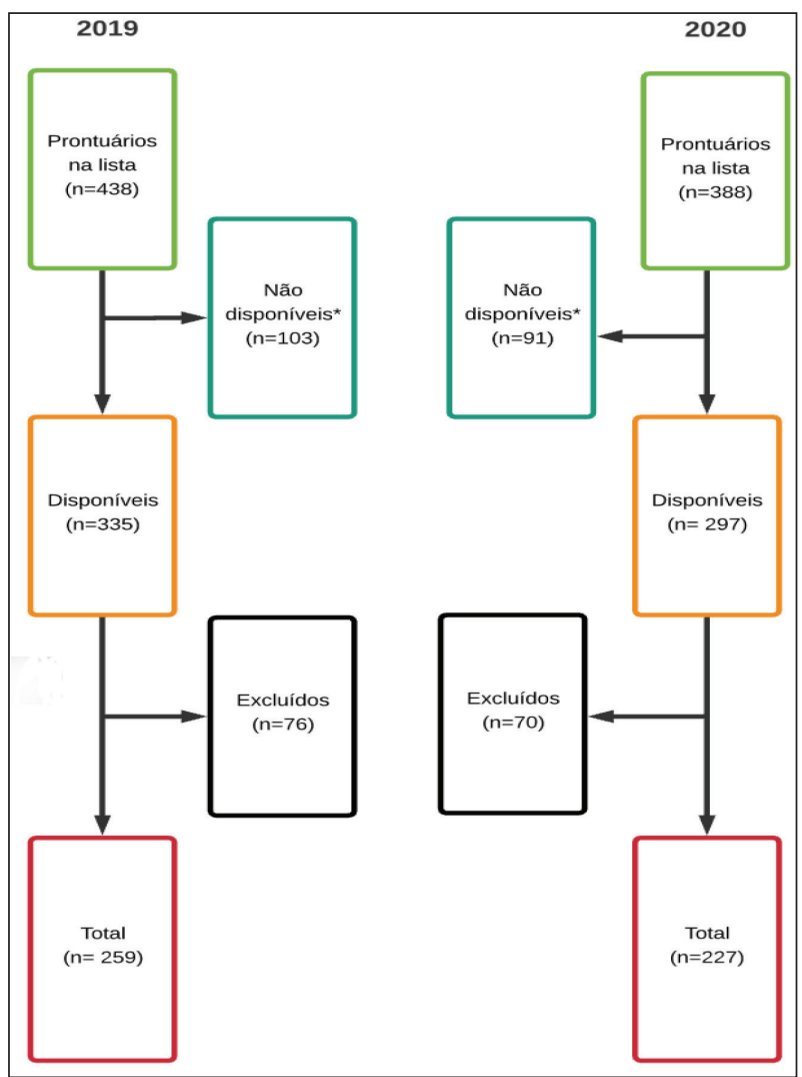

Fonte: UNACON/ OSID (2019-2020).

Legenda: * em uso em outros setores do hospital ou no arquivo histórico.

Tabela 1 -Dados sociodemográficos dos pacientes entre março e agosto de 2019 e 2020

\begin{tabular}{|c|c|c|}
\hline & $\begin{array}{c}2019 \\
(n=259)\end{array}$ & $\begin{array}{c}2020 \\
(n=227)\end{array}$ \\
\hline & $\mathrm{n}(\%)$ & $n(\%)$ \\
\hline \multicolumn{3}{|l|}{ SEXO } \\
\hline Feminino & $186(71 \%)$ & $163(71 \%)$ \\
\hline Masculino & $73(28 \%)$ & $64(28 \%)$ \\
\hline \multicolumn{3}{|l|}{ FAIXA ETÁRIA } \\
\hline $19-40$ anos & $21(8 \%)$ & $20(9 \%)$ \\
\hline $41-60$ anos & $122(47 \%)$ & $110(48 \%)$ \\
\hline Mais de 60 anos & $116(45 \%)$ & 97 (43\%) \\
\hline \multicolumn{3}{|c|}{ LOCAL DE RESIDÊNCIA } \\
\hline Salvador & 132 (51\%) & $108(48 \%)$ \\
\hline RMS & $17(7 \%)$ & $26(11 \%)$ \\
\hline TFD & $109(42 \%)$ & 93 (41\%) \\
\hline
\end{tabular}

Fonte: UNACON/ OSID (2019-2020).

Legenda: RMS - Região Metropolitana de Salvador; TFD - Tratamento Fora de Domicílio
Figura 2 - Número mensal de atendimentos entre março e agosto de 2019 e 2020.

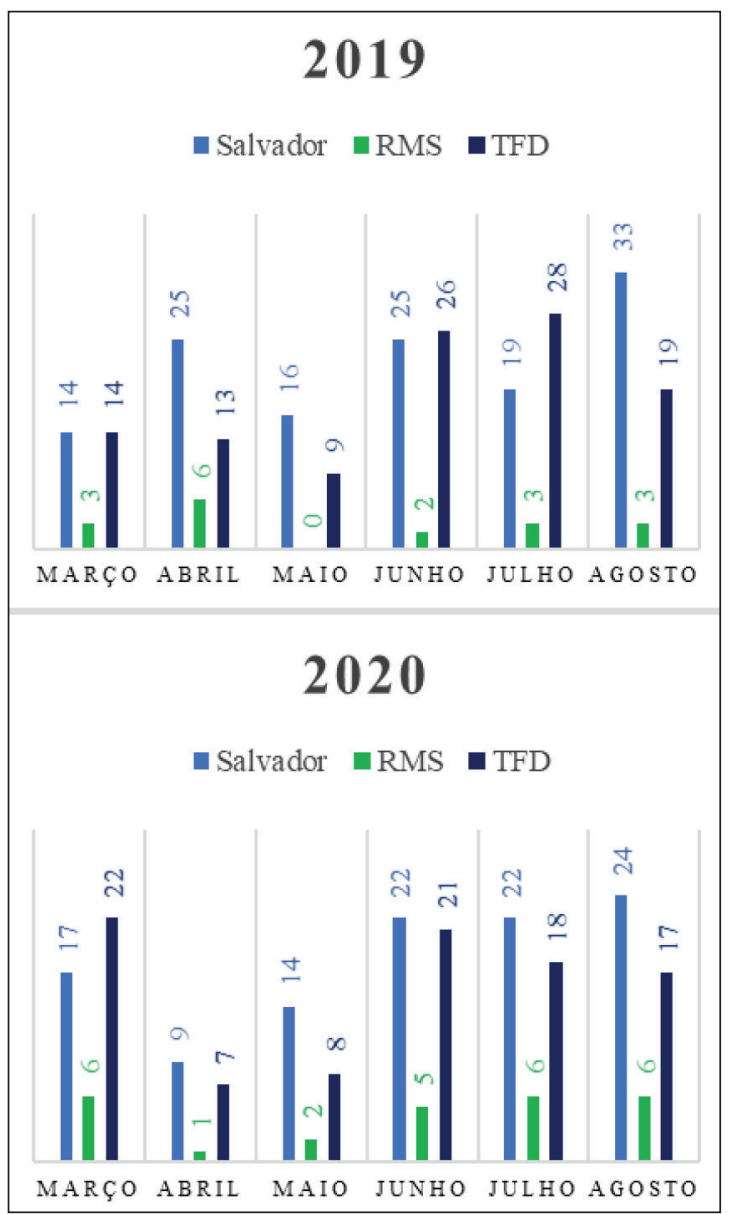

Fonte: UNACON/ OSID (2019-2020).

Além disso, calculou-se a média de dias em que os pacientes demoraram para realizar a primeira sessão de radioterapia, após a data do primeiro diagnóstico, presente na folha de atendimento do serviço de radioterapia, e foi possível ver que em 2019 esse tempo foi de 77 dias e em 2020, de 51 dias.

Os dados relacionados aos tipos de tumores mais frequentes no serviço e o estadiamento tumoral para os dois anos estão presentes nas Figuras 3 e 4, respectivamente. 
Figura 3 - Tipos de tumores mais frequentes entre março $e$ agosto de 2019 e mesmo período de 2020.

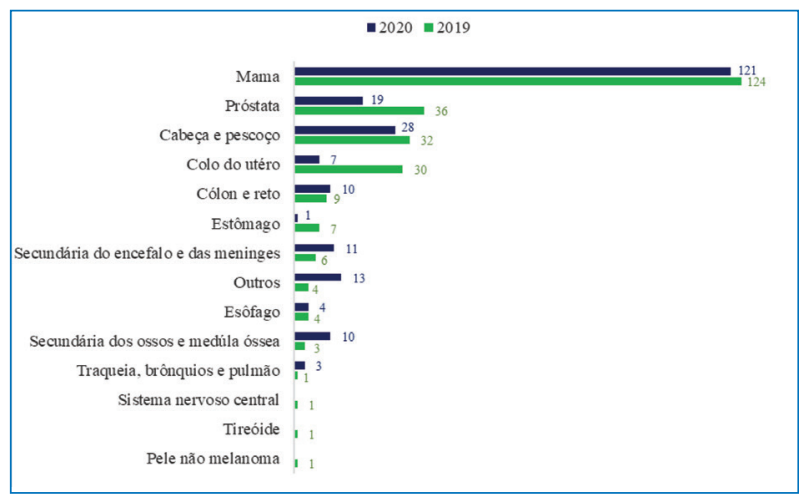

Fonte: UNACON/ OSID (2019-2020).

Figura 4 - Estadiamento tumoral entre março e agosto de 2019 e mesmo período de 2020.

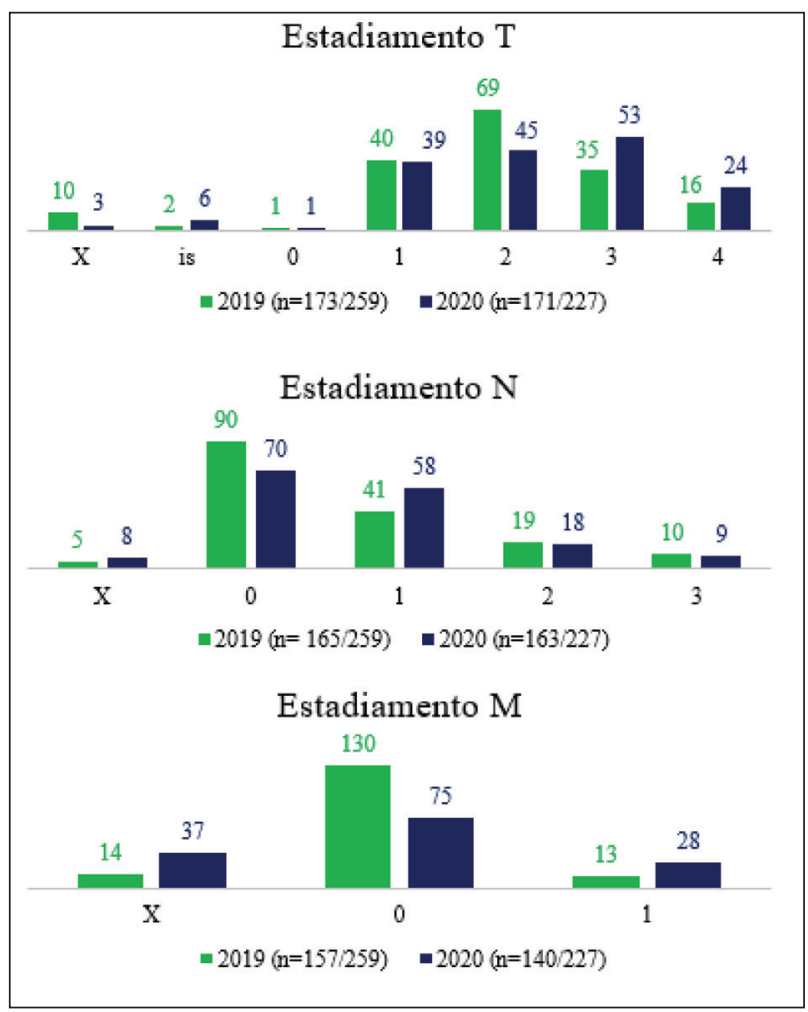

Fonte: UNACON/ OSID (2019-2020).

\section{DISCUSSÃO}

No presente estudo, pode-se observar que houve uma queda bruta de $12 \%$ no número de atendimentos de pacientes de primeiras consultas para o serviço de radioterapia, ao se comparar os seis primeiro meses da pandemia por COVID-19 com o mesmo período do ano anterior. Em um levantamento realizado pela Sociedade Brasileira de Radioterapia (SBRT) no ano de 2020, 6 em cada 10 serviços de radioterapia apresentaram, de ma- neira geral, queda no número de pacientes atendidos, tendo como principais causas a escolha pelo adiamento da realização radioterapia (46\%), medo por parte dos pacientes e familiares (38\%) e redução no número de diagnósticos (38\%) (SOCIEDADE BRASILEIRA DE RADIOTERAPIA, 2020). Por se tratar de análise de prontuários, não foi possível saber as causas que levaram à queda do número de pacientes no serviço estudado. Ainda, neste trabalho, foi observada, uma variação nos principais tipos de tumores atendidos e o estadiamento tumoral dos pacientes avaliados.

Apesar de mostrar uma variação no número de pacientes atendidos mensalmente de um ano para o outro, esses números não representam de forma fidedigna $o$ número de atendimentos realizados na unidade em questão, uma vez que alguns prontuários aparecem listados de forma repetida e ainda há casos em que o paciente aparece na lista, porém só realizou a radioterapia meses depois. É importante ressaltar que a busca de prontuário e coleta de dados foi realizada manualmente, por isso alguns prontuários não puderam ser encontrados, por estarem em uso em outros setores do ambulatório ou no arquivo histórico. Ainda assim, esse trabalho se mostra importante ao apontar indícios de como a pandemia pode ter afetado o tratamento antineoplásico no Estado da Bahia, bem para direcionar pesquisas futuras referentes ao tema abordado.

Em relação ao perfil sociodemográfico dos pacientes atendidos na radioterapia no período estudado, não houve diferença em relação ao sexo nos dois períodos escoIhidos, sendo a maioria dos pacientes do sexo feminino, representada por $71 \%$ da população. Pacientes com idade entre 19 e 40 anos representaram apenas $9 \%$ dos pacientes, sendo os demais classificados quase igualmente nas faixas etárias de 41 até 60 anos e acima de 60 anos com $47 \%$ e $45 \%$, respectivamente, no ano de 2019. Em 2020, houve uma discreta redução de $2 \%$ na população com mais de 60 anos, que é considerada a população de risco para COVID-19. O estudo de Nunes et al. (2020), realizado no Brasil, evidenciou que boa parte da população em processo de envelhecimento apresenta comorbidades relevantes para o agravamento e desfecho da COVID-19. Em estudo realizado no primeiro trimestre da pandemia, com dados coletados do site Portal do COVID-19 (SIVEP-Gripe e e-SUS-VE base de dados combinadas), de uma amostra de 67.180 pacientes com COVID-19, 65,5\% tinham mais de 50 anos de idade, bem como $85 \%$ (20.916 de 24.519) dos que vieram a óbito se encontravam nessa faixa etária (SOUZA et al., 2020). A divulgação de dados semelhantes a esses pode ter justificado a redução, mesmo que pequena, de indivíduos na faixa etária de maior risco, buscando atendimento oncológico.

Mesmo apresentando uma similaridade nos números absolutos de atendimentos nos dois períodos, é possível observar que, no mês de março de 2019, o número de pacientes $(n=31)$ foi inferior ao ano de $2020(n=45)$, o que não seria o esperado. Todavia, esse fato pode ser 
explicado pela ausência de atendimento no período de carnaval (ocorrido em março de 2019) e mais uma semana em que $o$ atendimento foi suspenso por motivos internos do serviço. Porém, no mês abril de 2020, é possível perceber uma queda de $61 \%$ no número de atendimentos, sendo que não houve suspensão do serviço oncológico, mantendo-se o fluxo padrão do atendimento do serviço de radioterapia no período, o que pode mostrar, de uma forma mais real, o impacto da pandemia em seu primeiro momento. Nos meses seguintes os números voltaram a se equiparar nos dois períodos, havendo uma mudança no perfil de neoplasia maligna diagnosticada e estadiamento clínico, porém, sem alterar a quantidade de atendimentos. A não redução de número de atendimentos é uma informação positiva deste estudo.

A fim de conter o avanço do novo coronavírus, em 20 de março, o Governo do estado da Bahia, através do Decreto n-19.549 (BAHIA, 2020a), suspendeu o transporte coletivo intermunicipal, público e privado, nas modalidades regular, fretamento, complementar, alternativo e de vans, no município de Camaçari e, até junho de 2020, mais 314 municípios tinham o serviço de transporte coletivo intermunicipal suspenso (BAHIA, 2020b). Mesmo assim, o número de pacientes realizando tratamento fora de domicílio permaneceu próximo ao número de residentes da cidade de Salvador ( $n=93 ; n=108$, respectivamente), sendo contrário às expectativas do estudo, onde se esperava uma diminuição no número de pacientes realizando tratamento fora do domicílio e um aumento no quantitativo de pacientes residentes na capital. Porém, foi possível perceber um aumento de $53 \%$ no número de pacientes vindos da região metropolitana de Salvador. Uma possível explicação para isso seria o uso de carros particulares, o uso de transportes oferecidos pelos municípios para pacientes que realizam tratamento fora do domicílio e/ou uma estadia em Salvador, o que estimularia a manutenção das consultas por esses pacientes.

O câncer de mama se mostrou como a neoplasia maligna mais frequente tratada no serviço, tanto em 2019, quanto em 2020, com um número muito maior de casos em relação às outras neoplasias atendidas. Ao contrário do câncer de mama, o câncer de próstata e colo do útero, que ocupavam a segunda e quarta posição de neoplasias mais atendidas, exibiram uma redução de $47 \%$ e $76 \%$, respectivamente no ano de 2020 , em comparação a 2019. Juntamente a isso é possível notar um aumento de mais de $50 \%$ das neoplasias metastáticas. Seguindo as estimativas do Instituto Nacional de Câncer (INCA), no ano de 2020 para o estado da Bahia, o câncer de próstata aparecia com a maior taxa de incidência com 6.130 casos, enquanto o câncer de mama exibia uma estimativa de 3.460 casos, seguidos pelo câncer de colón e reto, colo do útero, traqueia, brônquios e pulmões. 0 câncer de cavidade oral, que se encaixa nos canceres de cabeça e pescoço, ao contrário do presente estudo onde aparece em segundo lugar entre as neoplasias mais prevalentes, ocupava o sétimo lugar na taxa de incidência das estimativas apresentadas (INSTITUTO NACIONAL DE CÂNCER, 2020).

Estudos longitudinais, com maiores períodos de avaliação, poderão trazer informações mais precisas em relação a essa mudança de perfil no tipo de neoplasia diagnosticada e se a mudança foi realmente ocasionada pela pandemia da COVID-19. No entanto, abre-se um questionamento em relação ao aumento do número de pacientes com metástase e neoplasias secundárias. É possível que esses pacientes tiveram maior possibilidade de acesso ao serviço, pelo fato de que pacientes com neoplasias incipientes preferiram aguardar uma melhora da situação da pandemia para buscar seu tratamento. Essa informação sobre a modificação com piora do estadiamento clínico dos pacientes atendidos pode ser mais bem avaliada através dos dados obtidos pela classificação de estadiamento TNM, onde T refere-se ao tamanho do Tumor, $\mathrm{N}$ significa ao acometimento dos linfonodos regionais e $\mathrm{M}$ metástase à distância.

O estadiamento tumoral no período estudado mostrou mudança, em comparação ao ano de 2019 e 2020, que foi percebida também nas neoplasias frequentes. Em relação ao $T$ foi possível ver uma diminuição do $T_{x}(-70 \%)$ e $\mathrm{T}_{2}(-34,7 \%)$ do ano 2019 para 2020, juntamente com um aumento do estadiamentos $\mathrm{T}_{3}(51,4 \%)$ e T4 (50\%). 0 $\mathrm{N}_{0}$ que é indicativo de não acometimento dos linfonodos regionais exibiu uma redução de $22,2 \%$ e um aumento do $N_{1}$ de $41,5 \%$, e do $N_{x}$ de $60 \%$. A maior diferença percebida entre as variáveis relacionadas ao estadiamento clínico aconteceu no índice relacionado à metástase, onde houve uma queda de $42,3 \%$ no $M_{0^{\prime}}$ que mostra ausência de metástase e um aumento de $115,4 \%$ no $M_{1}$, que indica a presença de metástase, o que também refletiu no aumento de mais de $50 \%$ das neoplasias secundárias, como mostrou o estudo. A quantidade de dados apresentados no estadiamento tumoral não corresponde ao valor total de cada ano $(2019=259 ; 2020=227)$, devido à ausência de informação nos prontuários do serviço de radioterapia ou por apresentar dados incompletos entre as variáveis que o compõem

Uma informação positiva obtida neste estudo se refere à diminuição do tempo médio de espera para início do atendimento radioterápico. A redução de $33,7 \%$ em dias é expressiva e com certeza foi importante para os pacientes que apresentavam estadiamento tumoral mais grave no período da pandemia. Essa redução pode ser, mais uma vez, resultado da liberação de vagas pelos pacientes com diagnósticos iniciais/precoces, possibilitando o atendimento mais rápido dos casos mais graves. A busca por essas causas deve ser objetivo de estudos futuros.

Foi escolhido o período entre março e agosto de 2020 como comparativo, a fim de observar as possíveis mudanças causadas pelo coronavírus logo após os decretos emitidos pelo governo estadual, colocando todo o estado da Bahia em situação de emergência pública, em decorrência da doença infecciosa viral, onde foram apresentadas medidas de distanciamento social, com o 
fechamento de ambientes que promoviam aglomeração, uma maior restrição de mobilidade pela proibição de circulação de meios de transportes coletivos intermunicipais e interestaduais, além do receio de se contaminar (BAHIA, 2020a).

Em um estudo realizado por Tian et al. em 2020, os autores afirmaram haver indicativos de que pacientes com câncer ou sobreviventes do câncer apresentam maior risco de serem infectados pelo novo coronavírus e também de desenvolverem formas mais graves da doença e até risco morte. Segundo o estudo de Zhang et al. (2020), além dos pacientes oncológicos serem mais vulneráveis ao novo coronavírus, os autores também revelam que o estágio do tumor pode afetar o curso da doença. Assim, esse trabalho se mostra relevante por tratar-se de uma primeira visão de como a COVID-19 pode ter afetado o serviço de cuidados em oncologia, quais características foram observadas, possibilitando uma análise para adoção de estratégias de cuidado e enfrentamento.

\section{CONCLUSÕES}

Com base nos resultados obtidos neste estudo, foi possível concluir que a pandemia do COVID-19, cujo início no Brasil ocorreu no mês de março de 2020 e se estende até o presente momento (setembro de 2021), causou um impacto quantitativo no atendimento do serviço de radioterapia da unidade estudada, apresentando uma diminuição de $12 \%$ no número de atendimentos realizados em 2020, em comparação com o mesmo período do ano de 2019. Além disso, houve uma piora do estadiamento tumoral, com um número maior de pacientes com acometimento dos linfonodos e com metástase, o que implicou também as neoplasias prevalentes, com o aumento de neoplasias secundárias no ano de 2020. Neste estudo, não houve uma diferença no percentual entre sexo e feminino e masculino no período estudado, porém constou-se uma redução de $2 \%$ na população acima de 60 anos, população de risco para o vírus Sars-Cov-2, sendo a maioria da amostra constituída de residentes da cidade de Salvador, tanto em 2019 quanto em 2020, porém com um aumento de $53 \%$ das pessoas vindas da Região Metropolitana de Salvador, no ano de 2020.

\section{AGRADECIMENTOS}

Daniella Souza Jesus, Ana Caroline Magalhães, Manoela Carrera e a toda equipe de arquivo da UNACON/OSID. Agradeço à Fundação de Amparo à Pesquisa do Estado da Bahia (FAPESB) pela concessão da bolsa de mestrado.

\section{REFERÊNCIAS}

BAHIA. Decreto № 19.529, de 16 de março de 2020a. Regulamenta, no Estado da Bahia, as medidas temporárias para enfrentamento da emergência de saúde pública de importância internacional decorrente do coronavírus. Salvador: Casa Civil, 2020. Disponível em: http://www. legislabahia.ba.gov.br/documentos/decreto-no-19529-de-16-de-marcode-2020. Acesso em: 12 mar. 2021.
BAHIA. Secretaria do Planejamento. Entenda as medidas de combate ao coronavírus na Bahia. Salvador: 2020b. Disponível em: http://www. seplan.ba.gov.br/2020/06/1582/Entenda-as-medias-de-combate-aocoronavirus-na-Bahia.html. Acesso em: 12 mar. 2021.

CHAN, J. J. et al. The impact of COVID-19 on and recommendations for breast cancer care: the singapore experience. Endocrine-Related Cancer, [s.I], v. 27, n. 9, p. 307-327, set. 2020. Disponível em: http:// dx.doi.org/10.1530/erc-20-0157. Acesso em: 20 mar. 2021.

FIALKA-MOSER, V. et al. Cancer rehabilitation: particularly with aspects on physical impairments. Journal of Rehabilitations Medicine, [s.I.], v. 35, p. 153-162. Jan. 2003.

INSTITUTO NACIONAL DE CÂNCER (INCA). Estimativa 2020: Bahia e Salvador - estimativa dos casos novos. Rio de Janeiro: INCA, 2020. Disponível em: https://www.inca.gov.br/estimativa/estado-capital/ bahia-salvador. Acesso em: 9 set. 2021.

INTERNATIONAL AGENCY FOR RESEARCH ON CANCER. Cancer Today. 2020. Disponível em: https://gco.iarc.fr/today/data/factsheets/ cancers/39-All-cancers-fact-sheet.pdf. Acesso em: 20 abr. 2021.

NUNES, B. P. et al. Multimorbidade e população em risco para COVID-19 grave no Estudo Longitudinal da Saúde dos Idosos Brasileiros. Cadernos de Saúde Pública, Rio de Janeiro, v. 36, n. 12, p. e00129620. DOI: 10.1590/0102-311X00129620.

SANTIAGO-PALMA, J.; PAYNE, R. Palliative care and rehabilitation. Cancer Supplement, [s.l.], v. 92, n. 4, p. 1049-1052, 2001.

SOUZA, W. M. S. et al. Epidemiological and clinical characteristics of the COVID-19 epidemic in Brazil. Nature Human Behavior, [s.I.], n. 4, p. 856-865, 2020. Disponível em: https://doi.org/10.1038/s41562-0200928-4. Acesso em: 20 abr. 2021.

SOCIEDADE BRASILEIRA DE RADIOTERAPIA. Impacto da pandemia COVID-19 nos serviços de radioterapia do Brasil. São Paulo: 2020. Disponível em: https://sbradioterapia.com.br/wp-content/ uploads/2021/03/SBRT_IMPACTO_COVID19.pdf. Acesso em: 12 mar. 2021.

TIAN, Y. et al. Cancer associates with risk and severe events of COVID -19: a systematic review and meta : analysis. International Journal of Cancer, New York, v. 148, n. 2, p. 363-374, 15 Aug. 2020. http://dx.doi. org/10.1002/ijc.33213.1863146.

WORLD HEALTH ORGANIZATION (WHO). Coronavirus disease (COVID-19). 2021a. Disponível em: https://www.who.int/emergencies/ diseases/novel-coronavirus-2019. Acesso em: 20 abr. 2021.

WORLD HEALTH ORGANIZATION (WHO). Cancer. 2021b. Disponível em: http://www.who.int/cancer/en/. Acesso em: 20 abr. 2021.

ZHANG, L. et al. Clinical characteristics of COVID-19-infected cancer patients: a retrospective case study in three hospitals within Wuhan, China. Annals of Oncology, Dordrecht, v. 31, n. 7, p. 894-901, July 2020. Disponível em: http://dx.doi.org/10.1016/j.annonc.2020.03.296. Acesso em: 20 abr. 2021

Submetido em: $19 / 11 / 2021$

Aceito em: 22/11/2021 